\title{
Anemia risk factors among people living with HIV across the United States in the current treatment era: a clinical cohort study
}

B. N. Harding ${ }^{1 *}$, B. M. Whitney ${ }^{1}$, R. M. Nance', S. A. Ruderman ${ }^{1}$, H. M. Crane' ${ }^{1}$ G. Burkholder ${ }^{2}$, R. D. Moore ${ }^{3}$, W. C. Mathews ${ }^{4}$, J. J. Eron ${ }^{5}$, P. W. Hunt ${ }^{6}$, P. Volberding ${ }^{6}$, B. Rodriguez ${ }^{7}$, K. H. Mayer ${ }^{8}$, M. S. Saag ${ }^{2}$, M. M. Kitahata', S. R. Heckbert ${ }^{1}$ and J. A. C. Delaney ${ }^{1}$

\begin{abstract}
Background: Anemia is common among people living with HIV infection (PLWH) and is associated with adverse health outcomes. Information on risk factors for anemia incidence in the current antiretroviral therapy (ART) era is lacking.

Methods: Within a prospective clinical cohort of adult PLWH receiving care at eight sites across the United States between 1/2010-3/2018, Cox proportional hazards regression analyses were conducted among a) PLWH free of anemia at baseline and b) PLWH free of severe anemia at baseline to determine associations between timeupdated patient characteristics and development of anemia (hemoglobin $<10 \mathrm{~g} / \mathrm{dL}$ ), or severe anemia (hemoglobin $<7.5 \mathrm{~g} / \mathrm{dL}$ ). Linear mixed effects models were used to examine relationships between patient characteristics and hemoglobin levels during follow-up. Hemoglobin levels were ascertained using laboratory data from routine clinical care. Potential risk factors included: age, sex, race/ethnicity, body mass index, smoking status, hazardous alcohol use, illicit drug use, hepatitis C virus (HCV) coinfection, estimated glomerular filtration rate (eGFR), CD4 cell count, viral load, ART use and time in care at CNICS site.

Results: This retrospective cohort study included 15,126 PLWH. During a median follow-up of 6.6 (interquartile range [IQR] 4.3-7.6) years, 1086 participants developed anemia and 465 participants developed severe anemia. Factors that were associated with incident anemia included: older age, female sex, black race, HCV coinfection, lower CD4 cell counts, VL $\geq 400$ copies/ml and lower eGFR.
\end{abstract}

Conclusion: Because anemia is a treatable condition associated with increased morbidity and mortality among PLWH, hemoglobin levels should be monitored routinely, especially among PLWH who have one or more risk factors for anemia.

Keywords: Anemia, Hemoglobin, Cohort, HIV

\footnotetext{
* Correspondence: hardingb@uw.edu

'Department of Medicine, University of Washington, 1959 NE Pacific Street, Health Sciences Building F-26, Box 357236, Seattle, WA 98195, USA

Full list of author information is available at the end of the article
}

C C The Author(s). 2020 Open Access This article is licensed under a Creative Commons Attribution 4.0 International License, which permits use, sharing, adaptation, distribution and reproduction in any medium or format, as long as you give appropriate credit to the original author(s) and the source, provide a link to the Creative Commons licence, and indicate if changes were made. The images or other third party material in this article are included in the article's Creative Commons licence, unless indicated otherwise in a credit line to the material. If material is not included in the article's Creative Commons licence and your intended use is not permitted by statutory regulation or exceeds the permitted use, you will need to obtain permission directly from the copyright holder. To view a copy of this licence, visit http://creativecommons.org/licenses/by/4.0/. The Creative Commons Public Domain Dedication waiver (http://creativecommons.org/publicdomain/zero/1.0/) applies to the data made available in this article, unless otherwise stated in a credit line to the data. 


\section{Background}

Anemia (hemoglobin $<10 \mathrm{~g} / \mathrm{dL}$ ) and severe anemia (hemoglobin $<7.5 \mathrm{~g} / \mathrm{dL}$ ) [1] are common among people living with HIV (PLWH) [2], and the prevalence of anemia increases with HIV disease severity [3, 4]. While estimates of anemia prevalence vary depending on factors including age, setting, HIV disease stage, use of antiretroviral therapy (ART), sex, and injection drug use status [2, 3], it is estimated that $18-32 \%$ of PLWH without AIDS and $48-85 \%$ of PLWH with clinical AIDS have anemia in the United States, compared to $15-17 \%$ of people living without HIV [3, 5]. Many of the aforementioned prevalence estimates date from before or early in the ART era before the current treatment initiation guidelines and improvements in HIV care [6].

Anemia is thought to be an independent prognostic indicator among PLWH, associated with HIV disease progression [2, 7]. Furthermore, previous studies have reported associations between anemia and mortality [4, 8-10], health-related quality-of-life, dementia [11], and treatment failure [12] among PLWH.

The causes of anemia among PLWH are multifactorial. Immune dysregulation during HIV infection can increase anemia risk through red blood cell destruction (hemolysis) or ineffective red blood cell production, which are influenced by infections of the spleen or circulatory system [13]. Blood loss among PLWH is not uncommon and may occur as a result of neoplastic disease or gastrointestinal lesions accompanying opportunistic infections. Additional mechanisms for HIV-related anemia include deficiencies of vitamin $\mathrm{B} 12$, folate, or iron [13].

The goal of this study was to estimate the association between patient characteristics and (a) risk of anemia, (b) risk of severe anemia, or (c) a lower hemoglobin level to identify risk factors for anemia among a large cohort of PLWH within the US in the modern HIV treatment era.

\section{Methods}

\section{Overview and setting}

This study included PLWH in care in the Centers for AIDS Research (CFAR) Network of Integrated Clinical Systems (CNICS) cohort during January 1, 2010 to March 31, 2018. The CNICS cohort has been described in detail elsewhere [14]. Briefly, CNICS is a dynamic prospective clinical cohort of more than 32,000 PLWH $\geq 18$ years of age receiving care at eight participating academic sites across the US. Comprehensive clinical data, including diagnoses, medications (including ART), laboratory test results, demographic information, and historical information, including ART use before enrollment, are collected through electronic medical records and other institutional data systems at each site and harmonized in the CNICS data repository. Medication data, including ART use, is entered into the electronic medical records by clinicians or prescription fill/ refill data are uploaded directly from Pharmacy Systems and verified through medical record review. Patient reported measures and outcomes data (PROs) are collected in the CNICS assessment as part of routine clinical care appointments $[15,16]$. However, sites varied in their year of PRO initiation, so it is available for the majority but not all CNICS participants. The CNICS clinical assessment of PROs includes measures of smoking, alcohol use and illicit drug use. Participants entered the present study on January 1, 2010 or the earliest date that they had been enrolled in CNICS for 6 months, whichever came later (baseline). Additional exclusions were made for participants without PRO data, or for those with less than two available hemoglobin lab values during the study years (Fig. 1). Informed consent was obtained from all participants to be part of CNICS and institutional review boards at each site approved CNICS protocols.

\section{Participant characteristics}

Characteristics that were analyzed as potential risk factors for anemia, severe anemia or a lower hemoglobin level included the following characteristics analyzed at baseline: age, sex, race/ethnicity, BMI (closest available BMI, categorized as $<18.5,18.5-<25,25-<30, \geq 30 \mathrm{~kg} /$ $\mathrm{m}^{2}$ ), smoking status (never, former, current), hazardous alcohol use (a score of $\geq 4$ for women or $\geq 5$ for men on the Alcohol Use Disorders Identification Test (AUDITC) scale [17]) illicit drug use (use of cocaine/crack, methamphetamines/crystal and/or illicit opioids, using the Alcohol, Smoking and Substance Involvement Screening Test (ASSIST) [18]). In addition, the following characteristics were analyzed as potential time-updated risk factors: hepatitis $\mathrm{C}$ virus $(\mathrm{HCV})$ coinfection, kidney function measured using estimated glomerular filtration rate (eGFR, categorized as $<30,30-59$, or $\geq 60 \mathrm{~mL} / \mathrm{mi}$ nute $/ 1.73 \mathrm{~m}^{2}$ ), CD4 cell count (categorized as $\geq 500$, 350-499, 200-399, 100-199 or $<100$ cells $/ \mathrm{mm}^{3}$ ), detectable viral load (VL, $\geq 400$ copies $/ \mathrm{ml}$ ), treatment with ART and time in care at CNICS site, defined as time from enrollment in CNICS until the last available CNICS activity (either last lab date or last visit). In addition, we adjusted for CNICS site. All covariates were selected a priori, based on review of the literature and clinical knowledge.

\section{Outcome ascertainment}

Hemoglobin levels, expressed in grams per deciliter (g/ $\mathrm{dL}$ ), were ascertained using inpatient and outpatient laboratory data obtained as part of routine clinical care. Outcomes included incident anemia (the first hemoglobin 


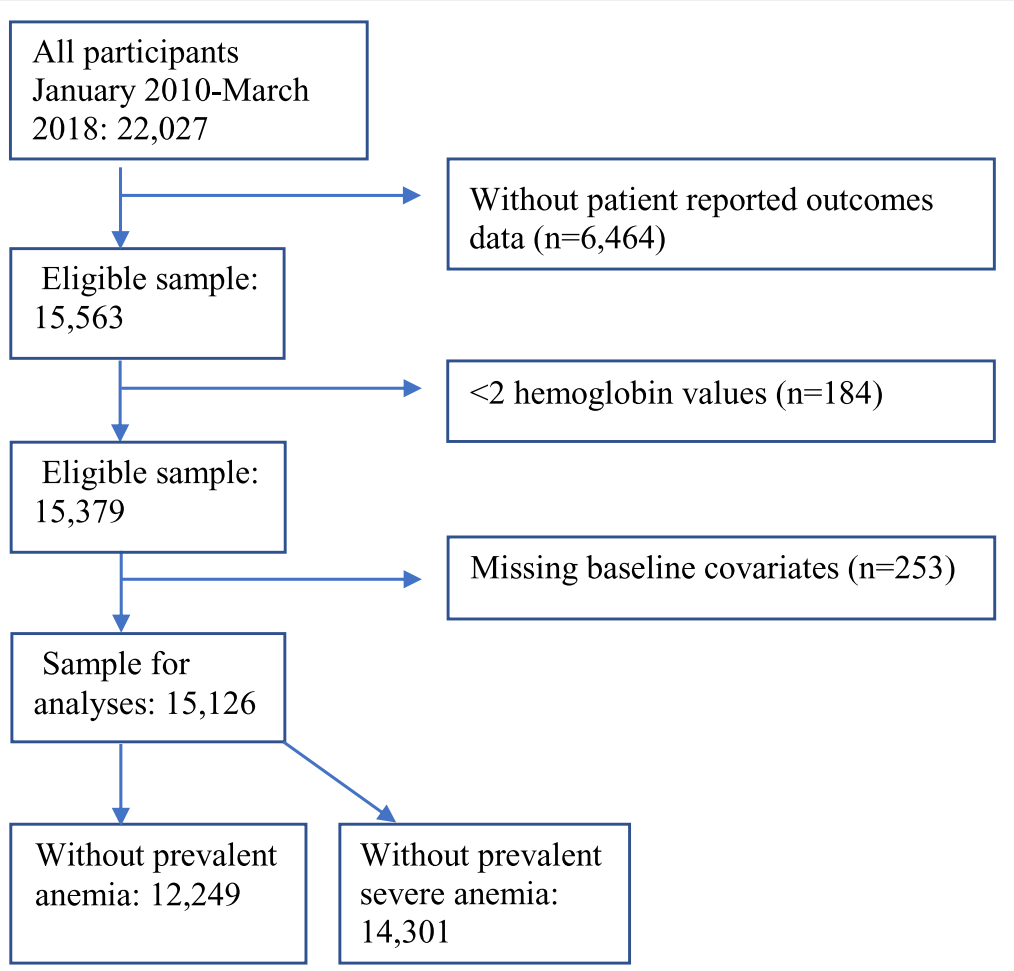

Fig. 1 Flow chart for inclusion/exclusion criteria

measure below $10 \mathrm{~g} / \mathrm{dL}$ ), incident severe anemia (the first hemoglobin measure below $7.5 \mathrm{~g} / \mathrm{dL}$ ), and changes in hemoglobin level. Additional interest lay in investigating associations between patient characteristics and chronic anemia, defined as anemia lasting for at least 6 months, identified using hemoglobin lab results on two separate occasions at least 6 months apart which were consistently in the anemic range without any additional hemoglobin values above the anemia range during that six-month period.

\section{Statistical analysis}

Two multivariable Cox proportional hazards regression analyses were conducted. First, among the subset of PLWH who did not have a history of anemia at baseline, associations were measured between risk factors and development of anemia. Second, among the subset of participants who did not have a history of severe anemia at baseline, associations were measured between risk factors and development of severe anemia. Participants were censored at (a) the time they developed anemia (for the incident anemia analyses) or at the time they developed severe anemia (for incident severe anemia analyses), (b) at the time of last activity in CNICS, (c) at the time of death or (d) at the time of administrative censoring due to end of follow-up per site, whichever came first. The timescale for the models was time since baseline. In post hoc analyses, we estimated the risk of anemia for subgroups identified as having combinations of high risk characteristics and tested for interaction on a multiplicative scale.

Linear mixed effects models were used to examine the associations between patient characteristics and hemoglobin levels among all PLWH. Mixed-effects models utilize random intercepts and slopes for time at the participant level to handle irregular patterns of repeated measures over follow-up [19].

\section{Sensitivity analysis}

In a sensitivity analysis, we assessed associations between patient characteristics and chronic anemia (defined as anemia lasting for at least 6 months) using a Cox proportional hazards regression model among the population who were anemia-free at baseline. Because factors such as severe bleeding, blood donation and blood transfusion could impact hemoglobin levels at a given time point and are difficult to capture, this sensitivity analysis allowed us to describe associations among those with chronic anemia.

All analyses were performed using Stata version 14.2.

\section{Results}

There were 15,126 PLWH who were included in these analyses (Fig. 1). A total of 12,249 (81\%) did not have a history of anemia at baseline, and 14,301 (95\%) did not have a history of severe anemia at baseline. Participants 
had a median of 14 hemoglobin values measured during a median follow-up of 6.6 (interquartile range [IQR] 4.3-7.6) years. Table 1 provides baseline characteristics for all study participants, as well as characteristics for the 1086 participants who developed anemia and 465 participants who developed severe anemia during follow-up. For the overall population, the median age of study participants was 44 (IQR 35-51) years at baseline, $17 \%$ were female, $17 \%$ were co-infected with $\mathrm{HCV}$, and $80 \%$ were using ART.

The incidence of anemia was $1.95 / 100$ person-years and the incidence of severe anemia was $0.68 / 100$ person-years. Characteristics associated with incident anemia included older age, female sex, black race/

Table 1 Baseline demographic and clinical characteristics of PLWH in care at sites across the United States overall and by anemia status $^{a}$

\begin{tabular}{|c|c|c|c|}
\hline Participant characteristics & $\begin{array}{l}\text { All participants } \\
(N=15,126)\end{array}$ & $\begin{array}{l}\text { Developed anemia } \\
\text { during follow-up } \\
\text { ( } n=1086 \text { ) }\end{array}$ & $\begin{array}{l}\text { Developed severe } \\
\text { anemia during } \\
\text { follow-up ( } n=465 \text { ) }\end{array}$ \\
\hline Age (median, IQR) & $44(35-51)$ & $46(39-53)$ & $46(39-53)$ \\
\hline Female & $2620(17)$ & $303(28)$ & $156(34)$ \\
\hline \multicolumn{4}{|l|}{ Race/ethnicity } \\
\hline White & $6712(44)$ & $411(38)$ & $148(32)$ \\
\hline Black & $5560(37)$ & $529(49)$ & $243(52)$ \\
\hline Hispanic & $2145(14)$ & $108(10)$ & $56(12)$ \\
\hline Other/missing & $709(5)$ & $38(3)$ & $18(4)$ \\
\hline Years in CNICS at baseline (median, IQR) & $1.1(0.5-6.0)$ & $2.2(0.5-7.0)$ & $3.0(0.5-7.7)$ \\
\hline Viral load $\geq 400$ copies/ml & $3314(22)$ & $338(31)$ & $170(37)$ \\
\hline \multicolumn{4}{|l|}{ CD4 count (cells/mm³) } \\
\hline$<100$ & $777(5)$ & $107(10)$ & $78(17)$ \\
\hline $100-199$ & $1236(8)$ & $99(9)$ & $64(14)$ \\
\hline $200-349$ & $2686(18)$ & $219(20)$ & $122(26)$ \\
\hline $350-499$ & $3323(22)$ & $233(22)$ & $79(17)$ \\
\hline$\geq 500$ & $7104(47)$ & $428(39)$ & $122(26)$ \\
\hline Hepatitis C virus coinfection & $2556(17)$ & $321(30)$ & $146(31)$ \\
\hline \multicolumn{4}{|l|}{ Kidney function (eGFR) } \\
\hline$<30$ & $164(1)$ & $25(2)$ & $23(5)$ \\
\hline $30-59$ & $620(4)$ & $51(5)$ & $34(7)$ \\
\hline$\geq 60$ & $14,342(95)$ & $1010(93)$ & $408(88)$ \\
\hline \multicolumn{4}{|l|}{ BMI $\left(\mathrm{kg} / \mathrm{m}^{2}\right)$} \\
\hline$<18.5$ & $352(2)$ & $24(2)$ & $20(4)$ \\
\hline 18.5 to $<25.0$ & $6494(43)$ & $472(44)$ & $207(44)$ \\
\hline 25.0 to $<30.0$ & $5199(34)$ & $339(31)$ & $128(28)$ \\
\hline$\geq 30.0$ & $3081(21)$ & $251(23)$ & $110(24)$ \\
\hline ART use & $12,070(80)$ & 821 (76) & $351(75)$ \\
\hline $\mathrm{MCV}(\mathrm{fL})$ & $91(87-95)$ & $91(86-96)$ & $90(85-96)$ \\
\hline \multicolumn{4}{|l|}{ Smoking status } \\
\hline Never & $6211(41)$ & $408(38)$ & $188(41)$ \\
\hline Former & $3492(23)$ & $195(18)$ & $80(17)$ \\
\hline Current & $5423(36)$ & $483(44)$ & $197(42)$ \\
\hline Hazardous alcohol use $\mathrm{b}^{\mathrm{b}}$ & $4239(28)$ & 244 (22) & $110(24)$ \\
\hline |llicit drug use ${ }^{c}$ & $2283(15)$ & $165(15)$ & $64(14)$ \\
\hline
\end{tabular}

${ }^{a}$ Baseline was defined as the earliest date during January 1, 2010- March 31, 2018 that a person had 6+ months in CNICS care

${ }^{b}$ Hazardous alcohol use defined a score of $\geq 4$ for women or $\geq 5$ for men on the AUDIT-C scale

' Use of cocaine/crack, methamphetamines/crystal, and/or illicit opioids

Abbreviations: ART antiretroviral therapy, BMI body mass index, eGFR estimated glomerular filtration rate, MCV mean corpuscular volume PLWH people living with HIV 
ethnicity, HCV coinfection, lower CD4 cell count, higher viral load, and lower eGFR, indicative of poor kidney function (Table 2). Risk factors associated with severe anemia were similar to those for anemia with the exception of older age and black race/ethnicity (Table 2). In post hoc analyses, we estimated the risk of anemia for subgroups identified as having combinations of high risk characteristics, including CD4 $<100$, female sex, and coinfection with HCV. Among women, CD4 $<100$ as compared with $\mathrm{CD} 4 \geq 500$ was associated with a HR for incident anemia of 10.64 (95\% CI 8.41, 13.47), while for men the HR was 4.11 (2.60, 6.52); $p$-value for low CD4-

Table 2 Factors associated with developing anemia or severe anemia in multivariable adjusted analyses among those free of anemia $(n=12,249)$ or among those free of severe anemia $(n=14,301)$ at baseline

\begin{tabular}{|c|c|c|}
\hline Participant characteristics & $\begin{array}{l}\text { Anemia } \\
n=12,249 \\
\text { Hazard ratio } \\
(95 \% \mathrm{Cl})\end{array}$ & $\begin{array}{l}\text { Severe anemia } \\
n=14,301 \\
\text { Hazard ratio } \\
(95 \% \mathrm{Cl})\end{array}$ \\
\hline Age per 10 years & $1.11(1.04,1.18)$ & $1.05(0.95,1.16)$ \\
\hline Female (ref male) & $2.45(2.12,2.82)$ & $2.09(1.69,2.58)$ \\
\hline \multicolumn{3}{|l|}{ Race/ethnicity (ref white) } \\
\hline Black & $1.19(1.03,1.39)$ & $0.83(0.65,1.05)$ \\
\hline Hispanic & $0.90(0.72,1.13)$ & $1.06(0.76,1.47)$ \\
\hline Other/missing & $0.99(0.71,1.39)$ & $1.13(0.69,1.86)$ \\
\hline Hepatitis C virus coinfection & $1.79(1.56,2.05)$ & $1.62(1.31,1.99)$ \\
\hline
\end{tabular}

CD4 cell count (cells/mm3) (ref $\geq 500)$

\begin{tabular}{lll} 
350-499 & $1.38(1.16,1.65)$ & $2.23(1.68,2.97)$ \\
200-349 & $1.67(1.39,2.02)$ & $2.39(1.78,3.22)$ \\
100-199 & $2.83(2.28,3.62)$ & $3.89(2.77,5.45)$ \\
$<100$ & $8.40(6.85,10.51)$ & $15.53(11.56,20.92)$ \\
Viral load $\geq 400$ copies/ml & $1.43(1.23,1.64)$ & $1.86(1.48,2.34)$ \\
Kidney function (eGFR) (ref > 60) & \\
30-60 & $2.35(1.91,2.89)$ & $2.67(1.97,3.63)$ \\
$<30$ & $17.84(13.94,24.02)$ & $23.01(17.85,29.68)$ \\
ART use & $0.92(0.80,1.05)$ & $1.00(0.81,1.23)$ \\
BMI (ref 18.5-<25) & & \\
$<18.5$ & $0.89(0.59,1.32)$ & $1.23(0.77,1.96)$ \\
25.0 to <30.0 & $0.88(0.77,1.02)$ & $0.73(0.58,0.91)$ \\
$\geq 30.0$ & $1.02(0.87,1.20)$ & $0.92(0.72,1.17)$ \\
Smoking status (ref never) & & \\
Former & $0.89(0.75,1.06)$ & $0.82(0.63,1.07)$ \\
Current & $1.08(0.94,1.25)$ & $0.90(0.72,1.12)$ \\
Hazardous alcohol use & $0.93(0.81,1.06)$ & $1.07(0.87,1.31)$ \\
Illicit drug use & $0.99(0.86,1.14)$ & $0.99(0.77,1.21)$ \\
\hline
\end{tabular}

${ }^{a}$ Hazardous alcohol use defined a score of $\geq 4$ for women or $\geq 5$ for men on the AUDIT-C scale

${ }^{b}$ Use of cocaine/crack, methamphetamines/crystal, and/or illicit opioids Abbreviations: ART: antiretroviral therapy; BMI: body mass index; eGFR: estimated glomerular filtration rate sex interaction <0.001; Supplemental Table 1). Among those with $\mathrm{HCV}$ coinfection, CD $4<100$ was associated with a HR for incident anemia of 10.20 (95\% CI 8.01, 12.98), while for those without $\mathrm{HCV}$ coinfection, the HR was 5.01 (95\% CI 3.39, 7.42; p-value for low CD4$\mathrm{HCV}$ coinfection interaction $=0.001$; Supplemental Table 2).

Among the 12,249 PLWH who were free of anemia at baseline, 265 developed chronic anemia (lasting for at least 6 months), during follow-up for an incidence of $0.46 / 100$ person-years. For chronic anemia, associations with patient characteristics were similar to those in the primary analysis of anemia. (Table 3 ).

Average hemoglobin levels remained steady during follow-up; the median level was $14.3 \mathrm{~g} / \mathrm{dL}$ (IQR 13.115.2) at baseline and $14.3 \mathrm{~g} / \mathrm{dL}$ (IQR $13.1-15.3$ ) at the

Table 3 Factors associated with developing chronic anemia in analyses among those without anemia at baseline; $n=12,249$

\begin{tabular}{|c|c|c|}
\hline Participant characteristics & Hazard ratio & $95 \% \mathrm{Cl}$ \\
\hline Age per 10 years & 1.18 & $1.04,1.34$ \\
\hline Female (ref male) & 3.25 & $2.48,4.27$ \\
\hline \multicolumn{3}{|l|}{ Race/ethnicity (ref white) } \\
\hline Black & 1.96 & $1.43,2.68$ \\
\hline Hispanic & 1.29 & $0.82,2.04$ \\
\hline Other/missing & 1.12 & $0.54,2.33$ \\
\hline Hepatitis C virus coinfection & 1.76 & $1.32,2.36$ \\
\hline \multicolumn{3}{|c|}{ CD4 cell count (cells/mm3) (ref $\geq 500)$} \\
\hline $350-499$ & 1.10 & $0.77,1.56$ \\
\hline 200-349 & 1.93 & $1.40,2.67$ \\
\hline $100-199$ & 1.83 & $1.17,2.88$ \\
\hline$<100$ & 4.11 & $2.67,6.33$ \\
\hline Viral load $\geq 400$ copies $/ \mathrm{ml}$ & 1.86 & $1.48,2.34$ \\
\hline \multicolumn{3}{|c|}{ Kidney function (eGFR) (ref > 60) } \\
\hline $30-60$ & 1.31 & $0.72,2.37$ \\
\hline$<30$ & 5.40 & $2.35,12.42$ \\
\hline ART use & 0.99 & $0.73,1.35$ \\
\hline \multicolumn{3}{|l|}{ BMI (ref 18.5- < 25) } \\
\hline$<18.5$ & 0.75 & $0.31,1.85$ \\
\hline 25.0 to $<30.0$ & 0.85 & $0.64,1.14$ \\
\hline$\geq 30.0$ & 0.82 & $0.59,1.15$ \\
\hline \multicolumn{3}{|c|}{ Self-reported smoking (ref never) } \\
\hline Former & 0.82 & $0.57,1.16$ \\
\hline Current & 0.93 & $0.70,1.24$ \\
\hline Hazardous alcohol use ${ }^{a}$ & 0.86 & $0.66,1.12$ \\
\hline Illicit drug use ${ }^{b}$ & 0.87 & $0.64,1.18$ \\
\hline
\end{tabular}

${ }^{a}$ Hazardous alcohol use defined a score of $\geq 4$ for women or $\geq 5$ for men on the AUDIT-C scale

${ }^{\mathrm{b}}$ Use of cocaine/crack, methamphetamines/crystal, and/or illicit opioids Abbreviations: $A R T$ antiretroviral therapy, BMI body mass index, eGFR estimated glomerular filtration rate 
last available measurement per person. Characteristics that were associated with having a lower hemoglobin level measured during follow-up time included: older age, female sex, non-white race/ethnicity, HCV coinfection, lower CD4 cell count, higher VL, lower eGFR, ART use, illicit drug use and $\mathrm{BMI}<18.5$; conversely, BMI between 25 to $<30$ or $\mathrm{BMI} \geq 30$ was associated with higher hemoglobin overtime. Other factors associated with a higher hemoglobin level included being a current smoker and reporting hazardous alcohol use (Table 4).

\section{Discussion}

Anemia is an important clinical condition among PLWH. In this study of 15,126 PLWH in care in the current treatment era (2010 and after) in the US, we observed that factors including older age, female sex, black race, $\mathrm{HCV}$ coinfection, lower CD4 cell counts, VL $\geq 400$ copies/ml and lower eGFR were associated with an increased risk of anemia.

Lower CD4 cell count has been previously identified as a risk factor for anemia. The present study confirmed these findings, however we report much larger increased risks of anemia $(\mathrm{HR}=8.40,95 \% \mathrm{CI}: 6.85,10.51)$ and severe anemia $(\mathrm{HR}=15.53,95 \% \mathrm{CI}: 11.56-20.92)$ among PLWH with CD4 cell counts $<100$ cells $/ \mathrm{mm}^{3}$ than have been previously reported [20-24]. Risks this large in magnitude suggest that additional screening and careful monitoring would potentially be useful among participants with CD4 levels $<100$ cells $/ \mathrm{mm}^{3}$. In addition, we found that those with CD4 levels $<100$ who were female or who also had HCV coinfection were at an especially high risk of anemia.

Similar to the present study, previous work has found that comorbidities such as HCV infection $[25,26]$ and poor kidney function [27] are associated with anemia risk. Furthermore, the current recommended treatment for chronic $\mathrm{HCV}$ has known side effects of reduced hemoglobin levels and reversible anemia [25, 26]. Additionally, high BMI has been associated with iron [28] and vitamin B12 deficiencies [29] and a lower chance of recovery from anemia among PLWH [30]. However, we did not find strong evidence in the present study that $\mathrm{BMI}$ is associated with anemia risk.

Prior work has also shown that smoking leads to increased hemoglobin levels and a reduction in the detection of anemia because smoking reduces the oxygen carrying capacity of red blood cells, to which the body responds to by increasing hemoglobin levels as a means to counteract this reduction in oxygen carrying capacity [31]. However, limited research exists into potential associations between the use of other substances including alcohol or illicit drugs and anemia. We found that being a current smoker was associated with small increases in hemoglobin over time, but did not detect any measurable
Table 4 Characteristics associated with hemoglobin level over follow-up time in analyses (linear mixed-effect model); $N=15,126$

\begin{tabular}{|c|c|c|c|}
\hline Participant characteristics & Coeff $^{a}$ & $95 \% \mathrm{Cl}$ & $P$-value \\
\hline Age per 10 years & -0.01 & $-0.01,-0.01$ & $<0.001$ \\
\hline Female (ref male) & -1.68 & $-1.74,-1.62$ & $<0.001$ \\
\hline \multicolumn{4}{|l|}{ Race/ethnicity (ref white) } \\
\hline Black & -0.75 & $-0.80,-0.70$ & $<0.001$ \\
\hline Hispanic & -0.15 & $-0.21,-0.08$ & $<0.001$ \\
\hline Other/missing & -0.13 & $-0.23,-0.03$ & 0.011 \\
\hline Hepatitis C virus coinfection & -0.21 & $-0.25,-0.16$ & $<0.001$ \\
\hline \multicolumn{4}{|l|}{ CD4 cell count (cells/mm3) (ref $\geq 500)$} \\
\hline $350-499$ & -0.18 & $-0.19,-0.16$ & $<0.001$ \\
\hline $200-349$ & -0.39 & $-0.41,-0.37$ & $<0.001$ \\
\hline $100-199$ & -0.81 & $-0.83,-0.78$ & $<0.001$ \\
\hline$<100$ & -1.49 & $-1.52,-1.46$ & $<0.001$ \\
\hline Viral load $\geq 400$ copies $/ \mathrm{ml}$ & -0.09 & $-0.11,-0.07$ & $<0.001$ \\
\hline \multicolumn{4}{|l|}{ Kidney function (eGFR) (ref > 60) } \\
\hline $30-60$ & -0.15 & $-0.16,-0.13$ & $<0.001$ \\
\hline$<30$ & -1.11 & $-1.15,-1.07$ & $<0.001$ \\
\hline ART use & -0.03 & $-0.04,-0.01$ & $<0.001$ \\
\hline \multicolumn{4}{|l|}{ BMI (ref 18.5-<25) } \\
\hline$<18.5$ & -0.24 & $-0.37,-0.10$ & 0.001 \\
\hline 25.0 to $<30.0$ & 0.24 & $0.19,0.29$ & $<0.001$ \\
\hline$\geq 30.0$ & 0.16 & $0.10,0.22$ & $<0.001$ \\
\hline \multicolumn{4}{|l|}{ Self-reported smoking (ref never) } \\
\hline Former & 0.05 & $-0.01,0.11$ & 0.055 \\
\hline Current & 0.15 & $0.10,0.20$ & $<0.001$ \\
\hline Self-reported hazardous alcohol use $\mathrm{e}^{\mathrm{b}}$ & 0.13 & $0.09,0.18$ & $<0.001$ \\
\hline Self-reported illicit drug use $\mathrm{e}^{c}$ & -0.19 & $-0.24,-0.14$ & $<0.001$ \\
\hline \multicolumn{4}{|c|}{$\begin{array}{l}{ }^{a} \text { Coefficient is the mean difference per year in hemoglobin }(\mathrm{g} / \mathrm{dL}) \text { for each } \\
\text { variable after adjustment for all other covariates presented in the table } \\
{ }^{\mathrm{b}} \text { Hazardous alcohol use defined a score of } \geq 4 \text { for women or } \geq 5 \text { for men on } \\
\text { the AUDIT-C scale } \\
{ }^{\mathrm{C}} \text { Use of cocaine/crack, methamphetamines/crystal, and/or illicit opioids } \\
\text { Abbreviations: ART antiretroviral therapy, BMI body mass index, eGFR } \\
\text { estimated glomerular filtration rate }\end{array}$} \\
\hline
\end{tabular}

differences in anemia risk related to current smoking, alcohol or other substance use in the present study.

In the early treatment era, use of zidovudine (AZT), was a known cause of bone marrow suppression [32]. In more recent years, AZT use has decreased substantially as other, better-tolerated ART medications have become available. Though prior research has found that being on ART is associated with lower anemia incidence and severity for most PLWH [23, 33-35] due to the ability of ART to control HIV disease progression, we did not find an association between ART use and a reduction in anemia or severe anemia incidence in the present 
study. This may be explained by possible pro-anemic side effects of certain types of currently used ART regimens [36].

Strengths of this study include the large and geographically diverse study population, longitudinal data structure, and availability of information on behaviors including smoking, alcohol use, and illicit drug use. However, there are important limitations of this study, including our inability to classify anemia based on red cell indices, lack of information on hemoglobinopathies, nutritional deficiencies, chemotherapy or other medications with bone marrow toxicity, instances of severe bleeding, blood donation or blood transfusion, all of which could impact hemoglobin levels at a given time point, and the possibility of residual confounding due to the observational nature of the study. Additionally, we relied on self-report of smoking and substance use, which may be underreported. Finally, because this study was conducted among PLWH in care in the United States, our findings may not generalize to PLWH who are not in care or reside outside of the United States.

\section{Conclusions}

In conclusion, we found that factors including older age, female sex, black race, HCV coinfection, lower CD4 cell counts, VL $\geq 400$ copies/ml, and lower eGFR were associated with an increased risk of anemia. Because anemia is a treatable condition associated with increased morbidity and mortality among PLWH, clinicians should be aware of these risk factors. Hemoglobin levels should be monitored routinely, especially among PLWH who have one or more of the risk factors for anemia so that treatment can be initiated if deemed necessary.

\section{Supplementary information}

Supplementary information accompanies this paper at https://doi.org/10. 1186/s12879-020-04958-z.

Additional file 1: Table S1. Stratified table of anemia risk for CD4 $<100$ and sex.

Additional file 2: Table S2. Stratified table for anemia risk and CD4< 100 and HCV coinfection.

\begin{abstract}
Abbreviations
PLWH: People living with HIV; ART: Antiretroviral therapy; CFAR: Centers for AIDS Research; CNICS: CFAR Network of Integrated Clinical Systems; PRO: Patient reported measures and outcomes data; BMl: Body mass index; AUDIT-C: Alcohol Use Disorders Identification Test; ASSIST: Alcohol, Smoking and Substance Involvement Screening Test; HCV: Hepatitis C virus; eGFR: estimated glomerular filtration rate; VL: Viral load; IQR: Interquartile range; $A Z T$ : zidovudine
\end{abstract}

\section{Acknowledgements}

We would like to acknowledge all CNICS study participants and personnel for their contributions to this work.

\section{Authors' contributions}

BNH has conducted all analyses, contributed to methodologic approach, and written the manuscript, BMW and RMN contributed to data preparation and analysis, JACD contributed to the analysis, SRH, HMC and JACD contributed to conception and design, HMC, GB, RDM, WCM, JJE, PWH, BR, KHM, MSS and MMK contributed to data collection and BNH, BMW, RMN, SAR, HMC, GB, RDM, WCM, JJE, PWH, PV, BR, KHM, MSS, MMK, SRH and JACD contributed to the interpretation of data and critically revising the manuscript for important intellectual content. All authors read and approved the final manuscript.

\section{Funding}

This project was funded by R01HL126538-01A1/National Heart, Lung and Blood Institute. They provided an unrestricted grant and we are completely independent from the study sponsors. Dr. Harding is supported by a National Heart, Lung and Blood Institute grant T32HL007828.

\section{Availability of data and materials}

The data that support the findings of this study are available from the CNICS Research Coordinating Committee, but restrictions apply to the availability of these data, which were used under license for the current study, and so are not publicly available. Data are however available from the authors upon reasonable request and with permission of CNICS Research Coordinating Committee.

Ethics approval and consent to participate

Informed consent was obtained from all participants to be part of CNICS and institutional review boards at each site approved CNICS protocols.

\section{Consent for publication}

Not applicable.

\section{Competing interests}

BNH, BMW, RMN, SAR, RDM, WCM, KHM, MMK, SRH, JACD report grants from the NHLBI during the conduct of the study.

HMC reports grants from NHLBI during the conduct of the study and grants from the $\mathrm{NIH}$, ViiV and PCORI outside the submitted work.

GB reports grants from NHLBI during the conduct of the study, and other fees from Amgen Inc. outside of the submitted work.

JJE reports grants from the $\mathrm{NIH}$ during the conduct of the study and grants and personal fees from Gilead Sciences and Viiv and personal fees from Janssen and Merck outside of the submitted work.

PWH reports grants from NHLBI during the conduct of the study, personal fees from Gilead, Viiv and Janssen and non-financial support from Merck outside the submitted work.

PV reports grants from NHLBI during the conduct of the study and other fees from Merck outside the submitted work.

BR reports grants from NHLBI during the conduct of the study and personal fees from Gilead and ViiV outside the submitted work.

MMS reports grants from NHLBI and NAID during the conduct of the study and grants from Gilead, Merck and ViiV Healthcare outside the submitted work.

\section{Author details}

'Department of Medicine, University of Washington, 1959 NE Pacific Street, Health Sciences Building F-26, Box 357236, Seattle, WA 98195, USA. ${ }^{2}$ University of Alabama Birmingham, Birmingham, USA. ${ }^{3}$ Johns Hopkins University, Baltimore, USA. ${ }^{4}$ University of California San Diego, San Diego, USA. ${ }^{5}$ University of North Carolina, Chapel Hill, USA. ${ }^{6}$ University of California San Francisco, San Francisco, USA. ${ }^{7}$ Case Western Reserve University,

Cleveland, USA. ${ }^{8}$ Fenway Health Institute, Boston, USA.

Received: 19 July 2019 Accepted: 10 March 2020

Published online: 20 March 2020

\section{References}

1. Olafsdottir M, Skoog I, Marcusson J. Detection of dementia in primary care: the Linkoping study. Dement Geriatr Cogn Disord. 2000;11(4):223-9.

2. Redig AJ, Berliner N. Pathogenesis and clinical implications of HIV-related anemia in 2013. Hematol Am Soc Hematol Educ Program. 2013;2013:377-81. 
3. Belperio PS, Rhew DC. Prevalence and outcomes of anemia in individuals with human immunodeficiency virus: a systematic review of the literature. Am J Med. 2004;116(Suppl 7A):27S-43S.

4. Sullivan PS, et al. Epidemiology of anemia in human immunodeficiency virus (HIV)-infected persons: results from the multistate adult and adolescent spectrum of HIV disease surveillance project. Blood. 1998;91(1): 301-8.

5. Levine AM, et al. Prevalence and correlates of anemia in a large cohort of HIV-infected women: Women's interagency HIV study. J Acquir Immune Defic Syndr. 2001;26(1):28-35.

6. Miller J, et al. A randomized, double-blind study of gemfibrozil for the treatment of protease inhibitor-associated hypertriglyceridaemia. AIDS. 2002; 16(16):2195-200.

7. Kowalska JD, et al. Current hemoglobin levels are more predictive of disease progression than hemoglobin measured at baseline in patients receiving antiretroviral treatment for HIV type 1 infection. AIDS Res Hum Retrovir. 2007:23(10):1183-8.

8. Mocroft A, et al. Anaemia is an independent predictive marker for clinical prognosis in HIV-infected patients from across Europe. EuroSIDA study group. AIDS. 1999;13(8):943-50.

9. Moore RD, Keruly JC, Chaisson RE. Anemia and survival in HIV infection. J Acquir Immune Defic Syndr Hum Retrovirol. 1998;19(1):29-33.

10. Harris RJ, et al. Prognostic importance of anaemia in HIV type-1-infected patients starting antiretroviral therapy: collaborative analysis of prospective cohort studies. Antivir Ther. 2008;13(8):959-67.

11. McArthur JC, et al. Dementia in AIDS patients: incidence and risk factors. Multicenter AIDS Cohort Study. Neurology. 1993;43(11):2245-52.

12. Anude CJ, et al. Immuno-virologic outcomes and immuno-virologic discordance among adults alive and on anti-retroviral therapy at 12 months in Nigeria. BMC Infect Dis. 2013;13:113.

13. Kerkhoff $A D$, et al. Resolution of anaemia in a cohort of HIV-infected patients with a high prevalence and incidence of tuberculosis receiving antiretroviral therapy in South Africa. BMC Infect Dis. 2014;14:3860.

14. Kitahata MM, et al. Cohort profile: the centers for AIDS research network of integrated clinical systems. Int J Epidemiol. 2008;37(5):948-55.

15. Fredericksen $\mathrm{R}$, et al. Integrating a web-based patient assessment into primary care for HIV-infected adults. J AIDS HIV Res. 2012;4(2):47-55.

16. Crane HM, et al. Routine collection of patient-reported outcomes in an HIV clinic setting: the first 100 patients. Curr HIV Res. 2007;5(1):109-18.

17. Bush $\mathrm{K}$, et al. The AUDIT alcohol consumption questions (AUDIT-C): an effective brief screening test for problem drinking. Ambulatory care quality improvement project (ACQUIP). Alcohol use disorders identification test. Arch Intern Med. 1998;158(16):1789-95.

18. Group, W.A.W. The alcohol, smoking and substance involvement screening test (ASSIST): development, reliability and feasibility. Addiction. 2002;97(9): 1183-94.

19. Laird NM, Ware JH. Random-effects models for longitudinal data. Biometrics. 1982;38(4):963-74.

20. Agus Somia IK, et al. High levels of serum IL-6 and serum hepcidin and low CD4 cell count were risk factors of anemia of chronic disease in HIV patients on the combination of antiretroviral therapy. HIV AIDS (Auckl). 2019;11:133-9.

21. Gedefaw $L$, et al. Anemia and risk factors in HAART naive and HAART experienced HIV positive persons in south West Ethiopia: a comparative study. PLoS One. 2013;8(8):e72202.

22. Jin $Y$, et al. Prevalence of anaemia among HIV patients in rural China during the HAART era. Int J STD AIDS. 2017;28(1):63-8.

23. Petraro $P$, et al. Determinants of Anemia among human immunodeficiency virus-positive adults at care and treatment clinics in Dar Es Salaam, Tanzania. Am J Trop Med Hyg. 2016;94(2):384-92.

24. Shen $Y$, et al. Prevalence of anemia among adults with newly diagnosed HIV/AIDS in China. PLoS One. 2013;8(9):e73807.

25. Manns MP, et al. Peginterferon alfa-2b plus ribavirin compared with interferon alfa-2b plus ribavirin for initial treatment of chronic hepatitis $\mathrm{C}$ : a randomised trial. Lancet. 2001;358(9286):958-65.

26. Bodenheimer $\mathrm{HC} \mathrm{Jr}$, et al. Tolerance and efficacy of oral ribavirin treatment of chronic hepatitis C: a multicenter trial. Hepatology. 1997;26(2):473-7.

27. McClellan $W$, et al. The prevalence of anemia in patients with chronic kidney disease. Curr Med Res Opin. 2004;20(9):1501-10.

28. Zhao L, et al. Obesity and iron deficiency: a quantitative meta-analysis. Obes Rev. 2015;16(12):1081-93.
29. Arshad $M$, et al. Iron deficiency anemia and megaloblastic anemia in obese patients. Rom J Intern Med. 2017:55(1):3-7.

30. Ndlovu Z, Chirwa T, Takuva S. Incidence and predictors of recovery from anaemia within an HIV-infected south African cohort, 2004-2010. Pan Afr Med J. 2014;19:114.

31. Nordenberg D, Yip R, Binkin NJ. The effect of cigarette smoking on hemoglobin levels and anemia screening. JAMA. 1990;264(12):1556-9.

32. Meidani $\mathrm{M}$, et al. Prevalence, severity, and related factors of anemia in HIV/ AIDS patients. J Res Med Sci. 2012;17(2):138-42.

33. Semba RD, Shah N, Vlahov D. Improvement of anemia among HIV-infected injection drug users receiving highly active antiretroviral therapy. J Acquir Immune Defic Syndr. 2001;26(4):315-9.

34. Moore RD, Forney D. Anemia in HIV-infected patients receiving highly active antiretroviral therapy. J Acquir Immune Defic Syndr. 2002;29(1):54-7.

35. Semba RD, et al. Highly active antiretroviral therapy associated with improved anemia among HIV-infected women. AIDS Patient Care STDs. 2001;15(9):473-80.

36. Harding BN, Whitney BM, Nance RM, Crane HM, Burkholder G, Moore RD, Mathews WC, Eron JJ, Hunt PW, Volberding P, Rodriguez B, Mayer KH, Saag MS, Kitahata MM, Heckbert SR, Delaney JAC. Class of antiretroviral drugs and anemia risk in the current treatment era. bioRxiv. 2019. https://doi.org/10. $1101 / 674549$.

\section{Publisher's Note}

Springer Nature remains neutral with regard to jurisdictional claims in published maps and institutional affiliations.
Ready to submit your research? Choose BMC and benefit from:

- fast, convenient online submission

- thorough peer review by experienced researchers in your field

- rapid publication on acceptance

- support for research data, including large and complex data types

- gold Open Access which fosters wider collaboration and increased citations

- maximum visibility for your research: over $100 \mathrm{M}$ website views per year

At BMC, research is always in progress.

Learn more biomedcentral.com/submissions 